\title{
Achromatic Dye-type Polarizer for Paper White Reflective Liquid Crystal Displays
}

\author{
Noriaki Mochizuki ${ }^{\dagger 1}$, Takahiro Ishinabe ${ }^{\dagger 2}$ (member), Daichi Fujiwara ${ }^{\dagger 3}$, \\ Daisuke Nakamura $^{\dagger 3}$, Norio Koma ${ }^{\dagger 3}$, Hideo Fujikake ${ }^{\dagger 2}$ (member)
}

\begin{abstract}
We have developed the achromatic polarizer using novel dichroic pigments. We established a method to control the light absorption, dichroic ratio, and alignment direction of dichroic pigments and successfully achieved high transmittance uniformity in visible wavelength range in both parallel and crossed states. This polarizer results in development of the low power monochrome-type reflective liquid crystal display with high reflectivity, paper white and motion picture capability.
\end{abstract}

Keywords: Achromatic polarizer, Dichroic pigment, Liquid crystal display, Reflective LCD, Paper white.

\section{Introduction}

The development of bright reflective displays with low power consumption and motion image capabilities has been anticipated for next-generation display applications, such as e-book readers and large digital signs ${ }^{1-17)}$. Realization of a polarizer without wavelength dependency in both the parallel and crossed states, known as an "achromatic polarizer", is expected to lead to the development of reflective displays with high image quality, similar to that of printed paper.

However, conventional polarizers used in liquid crystal displays (LCDs) employ dichroic pigments, such as iodine or dichroic dye, and have problem of the wavelength dependence of their transmittance in the parallel and crossed states, as shown in Fig.1. For example, high-contrast type iodine-based polarizer becomes greenish yellow in the parallel state, and paperwhite type iodine-based polarizer becomes bluish in the crossed state.

The iodine-based polarizer has two dichroic pigments: $\mathrm{I}_{3}{ }^{-}+$polyvinyl alcohol (PVA) complex and $\mathrm{I}_{5}{ }^{-}+\mathrm{PVA}$ complex, with absorption peaks at $480 \mathrm{~nm}$ and $600 \mathrm{~nm}$, respectively; Figure 2 shows the influence of the complex on the wavelength dependence of the polarizer.

\footnotetext{
Received March 30, 2018; Revised July 5, 2018; Accepted August 23, 2018

$\dagger 1$ Nippon Kayaku Co., Ltd.

(Tokyo, Japan))

$† 2$ Tohoku University.

(Sendai, Japan)

$\dagger 3$ Polatechno Co., Ltd.

(Joetsu, Japan)
}

In general, the dichroic ratio of the dichroic pigment at $480 \mathrm{~nm}\left(\mathrm{I}_{3}{ }^{-}+\mathrm{PVA}\right.$ complex $)$ is lower than that at $600 \mathrm{~nm}$ ( $\mathrm{I}_{5}{ }^{-}+\mathrm{PVA}$ complex). In addition, the $\mathrm{I}_{5}{ }^{-}+\mathrm{PVA}$ complex has an unnecessary absorption at wavelengths less than 550 $\mathrm{nm}$. The low dichroic ratio at $480 \mathrm{~nm}$, and the

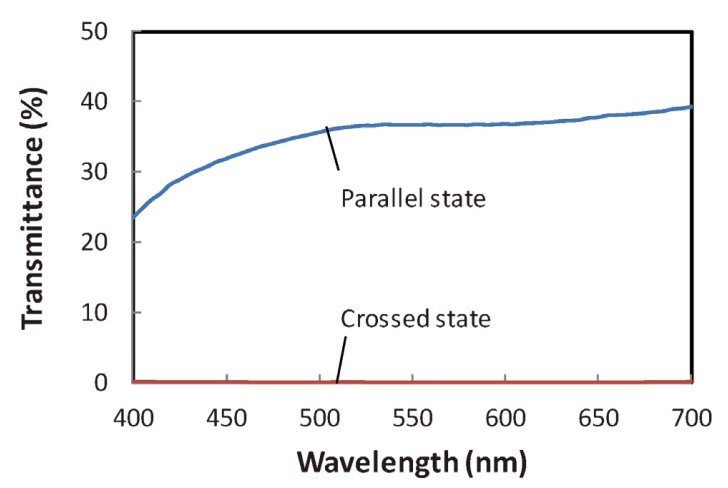

(a)

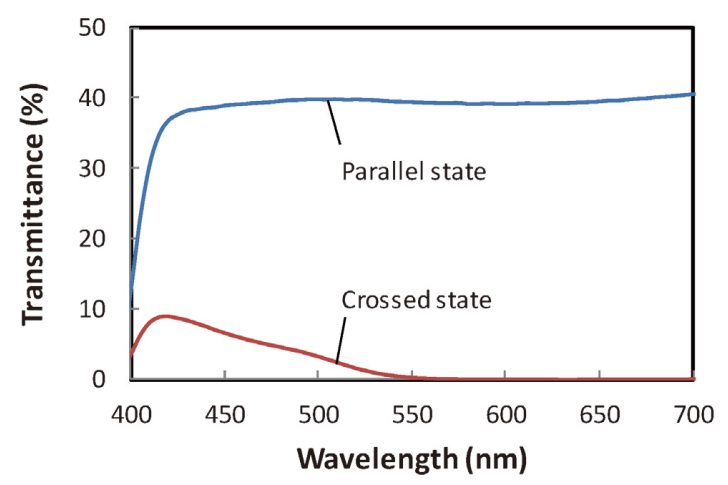

(b)

Fig.1 Wavelength properties of conventional Iodine-based polarizer. (a) High-contrast type polarizer and (b) paperwhite type polarizer. 


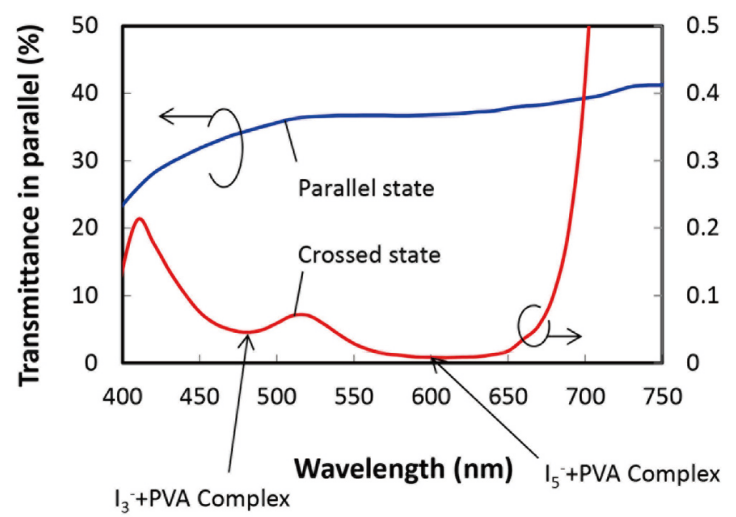

Fig.2 Influence of the complex on wavelength dependence of the iodine-based polarizer.

unnecessary absorption at less than $550 \mathrm{~nm}$, lead to low transmittance at $480 \mathrm{~nm}$ in the parallel state, for the high-contrast type polarizer with low transmittance in the crossed state, leading to the yellowish color in the parallel state. The otherwise paper-white type polarizer becomes bluish in the crossed state by the same reasoning. These problems greatly affect the image quality of LCDs, especially reflective displays using ambient light. Therefore, improvement of the wavelength dependency of polarizers is essential for development of high quality LCDs with low power consumption.

In this paper, we aim to realize an achromatic polarizer with no wavelength dependency in both the parallel and crossed states to achieve high quality and low power reflective LCDs using a dye-based polarizer. We investigated a method for controlling the absorbance and dichroic ratio of dichroic pigments and a manufacturing process to align each dichroic pigment uniformly. We also developed a prototype of a paperwhite monochrome-type reflective LCD with high reflectance and motion picture capability using the achromatic polarizer.

\section{Dichroic pigment for achromatic polarizer}

In general, dye-based polarizers contain several dichroic pigments, and each dichroic pigment has unnecessary light absorption in wavelength ranges outside the dominant wavelength. In addition, the dichroic ratio (degree of polarization) at the dominant wavelength is not uniform among dichroic pigments.

Figure 3 (a) shows the wavelength dependency of transmittance of the yellow, red and blue dyes used in the dye-based polarizer. The unnecessary absorption occurs in the short wavelength range ${ }^{18,19)}$.

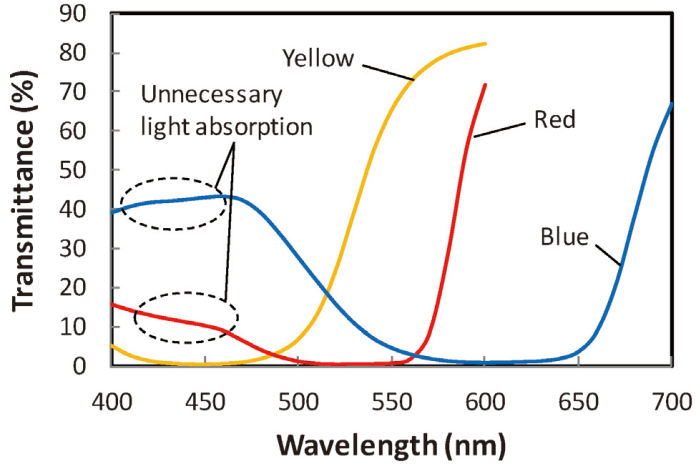

(a)

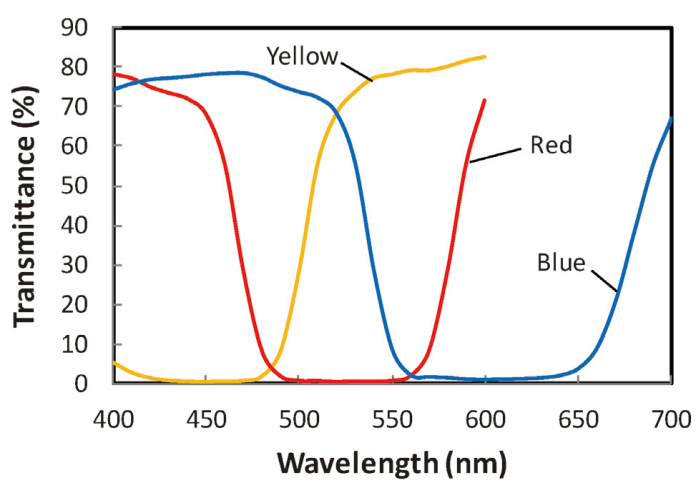

(b)

Fig.3 Wavelength dependency of (a) conventional dichroic pigments and (b) design concept of optimization of the light absorption and dichroic ratio of the new dichroic pigments in the dye-based polarizer.

The absorption of a polarizer is strongly dependent on the conjugate structure in the molecules ${ }^{20,21)}$. In particular, short conjugated structures, such as phenylazo-isogamma acid and benzoyl-isogamma acid, increase absorption in the short wavelength range.

To solve this problem and suppress light absorption occurring at wavelengths outside the dominant wavelength, we synthesized a new dichroic pigment that did not include a chemical structure with a conjugate in the substituent, and controlled the dichroic ratio of each dichroic pigment to be the same at each dominant wavelength. Figure 3 (b) shows the design concept of optimization of light absorption and the dichroic ratio of the new dichroic pigments in the dye-based polarizer.

Figure 4 shows the measured wavelength properties of the new dichroic pigment in the crossed state. We applied a single new dichroic pigment to a PVA film and stretched this in one direction. We obtained the polarizer by laminating a protective film (ex. tri-acetyl cellulose: TAC) on the surface of the PVA film. We also prepared polarizers using conventional dichroic pigments for comparison, and measured the wavelength dependence of the transmittance of all polarizers using a spectrophotometer. 


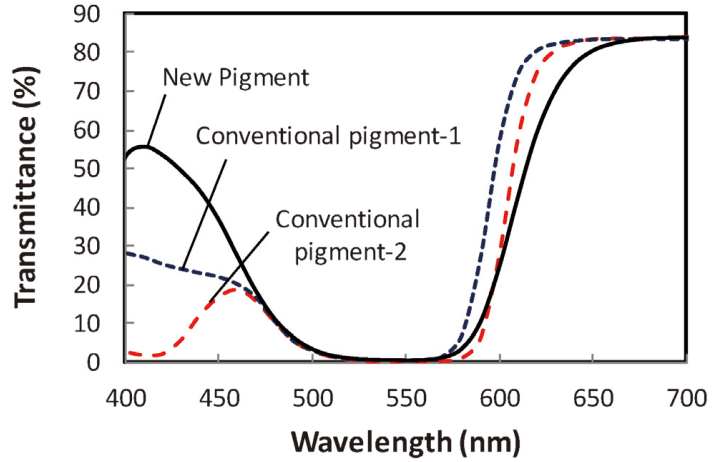

Fig.4 Measure results of wavelength dependency of dichroic pigments.

From Fig.4, we found that both conventional dichroic pigment 1 with a benzoyl-isogamma acid substituent and conventional dichroic pigment 2 with a phenyl-azoisogamma acid substituent exhibited strong absorption in the short wavelength range. In contrast, the new dichroic pigment exhibited less absorption in the short wavelength range.

This result shows that our new dichroic pigment suppresses light absorption at wavelengths less than $550 \mathrm{~nm}$ compared to the conventional dichroic pigments, and demonstrates an improved the dichroic ratio in the short wavelength range.

\section{Improvement of alignment uniformity of dichroic pigments}

Alignment control of dichroic pigments is also important for achieving a highly achromatic polarizer with a uniform dichroic ratio in the visible wavelength range. In general, the optimum conditions for the alignment process are different for each dichroic pigment because of their different chemical structures ${ }^{22-}$ 25). The migration of dichroic pigments in the thickness direction of the film also varies depending on the chemical structure; therefore, uniform stretching of the PVA film is important for improving the alignment uniformity of dichroic pigments in the thickness direction of the film.

We improved the method for comprising dichroic pigments and the crosslinker (boric acid). In general, PVA film can be highly orientated through stretching in a boric acid aqueous solution, therefore, we considered that uniform stretching of the PVA film can be achieved through a uniform boric acid distribution in the film.

In the manufacturing process of the dye-based polarizer, the component distributions of dichroic pigments and the crosslinker become heterogeneous due to the different inclusions in the PVA film. If the

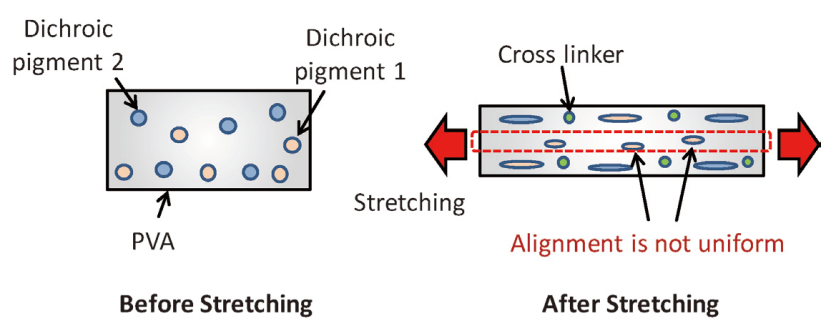

(a)

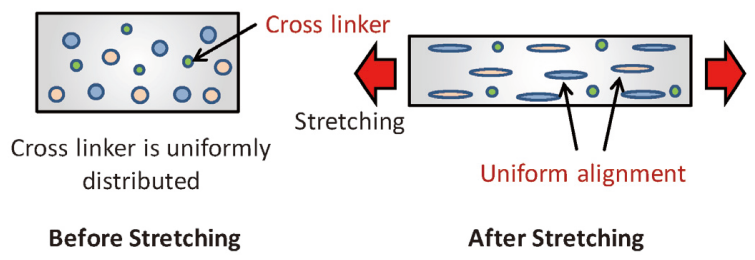

(b)

Fig.5 Stretching process of polarizers to improve the alignment of dichroic pigment. (a) Conventional method and (b) new method.

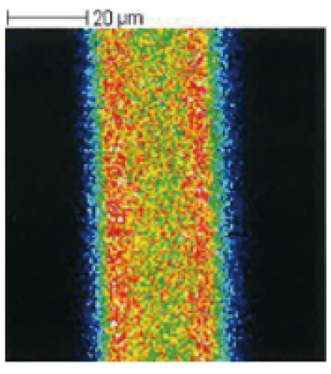

(a)

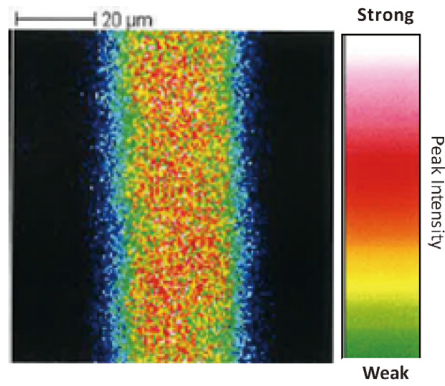

(b)
Fig.6 Boric acid content distribution in polarizer obtained by ToFSIMS analysis. (a) Conventional method and (b) new method.

Table 1 Dichroic ratio of dichroic pigment for several wavelength ranges.

\begin{tabular}{|c|c|c|c|}
\hline & $\begin{array}{c}\text { Pigment for } \\
430-480 \mathrm{~nm}\end{array}$ & $\begin{array}{c}\text { Pigment for } \\
520-580 \mathrm{~nm}\end{array}$ & $\begin{array}{c}\text { Pigment for } \\
580-640 \mathrm{~nm}\end{array}$ \\
\hline $\begin{array}{c}\text { Dichroic Ratio of } \\
\text { Conventional } \\
\text { pigment }\end{array}$ & 42 & 46 & 52 \\
\hline $\begin{array}{c}\text { Dichroic Ratio of } \\
\text { Improvement } \\
\text { pigment }\end{array}$ & 45 & 45 & 45 \\
\hline
\end{tabular}

component distribution is heterogeneous before stretching, the alignment uniformity of dichroic pigments will be degraded. We therefore improved the distribution of the crosslinker component and the alignment uniformity of dichroic pigments by impregnating the film with the dichroic pigment and crosslinked materials before stretching (see Fig.5).

Figure 6 shows the boric acid content distribution in the thickness direction of the polarizer measured by time-of-flight secondary ion mass spectrometry (ToFSIMS) analysis; Table 1 shows the maximum dichroic 
Table 2 Optical properties of polarizers.

\begin{tabular}{|c|c|c|c|c|c|c|c|c|}
\hline \multirow{2}{*}{ Sample } & \multirow{2}{*}{$\begin{array}{c}\text { Single } \\
\text { Transmittance } \\
\text { (\%) }\end{array}$} & \multirow{2}{*}{$\begin{array}{c}\text { Degree of Polarization } \\
(\%)\end{array}$} & \multicolumn{2}{|c|}{ Singlecolor } & \multicolumn{2}{|c|}{ Parallel nicol } & \multicolumn{2}{|c|}{ Cross nicol } \\
\hline & & & $a *_{-s}$ & $b^{*}-s$ & $a *-p$ & $b^{*}-p$ & $a^{*}-c$ & $b^{*}-c$ \\
\hline High Contrast lodine Polarizer & 42.09 & 99.99 & -1.37 & 4.15 & -2.36 & 7.62 & 0.02 & -0.07 \\
\hline Paper White lodine Polarizer & 44.86 & 97.87 & 0.32 & -2.79 & -1.00 & 0.96 & 19.56 & -38.73 \\
\hline \multirow{3}{*}{ New Achromatic Polarizer } & 39.43 & 99.99 & 0.01 & 0.48 & 0.09 & 0.72 & 0.50 & -0.92 \\
\hline & 44.92 & 87.46 & 0.12 & 0.12 & 0.64 & 0.10 & -0.89 & 0.34 \\
\hline & 50.02 & 75.27 & 0.25 & 0.31 & 0.76 & 0.67 & -0.07 & 0.01 \\
\hline
\end{tabular}

ratio of the dichroic pigment in each wavelength ranges. We found that boric acid, as the crosslinking agent, was uniformly distributed and our polarizer exhibited a uniform dichroic ratio in the visible wavelength range. In general, the PVA film is crosslinked using boric acid aqueous solution; however, if the PVA film stretches before impregnation of the crosslinked materials, the molecular density of the PVA increases, leading to crystallization of the film. As a result, the swell capacity of the PVA film decreases, and the boric acid aqueous solution does not penetrate into the inside of the PVA film; therefore, sufficient crosslinking cannot occur inside the PVA film in the conventional method. Our results thus confirm that impregnation of the PVA film with the dichroic pigment and crosslinked materials before stretching improves the uniformity of the dichroic ratio of the polarizers.

\section{Optical characteristics of achromatic polarizer}

Table 2 shows the single transmittance, degree of polarization and chromaticity of our new achromatic polarizer in the parallel and crossed states. The transmittance of the polarizers was measured using a spectrophotometer in both the parallel and crossed states, and the degree of polarization and chromaticity were calculated from the measurement results.

The single transmittance was defined as the luminous transmittance of a single polarizer. We fabricated achromatic polarizers with different transmittances by controlling the concentration of dichroic pigments: a high-transmittance type (single transmittance: $44.9 \%$ ) and a high-contrast type (single transmittance: $39.6 \%$ ). Table 2 also shows the optical properties of an achromatic polarizer with a single transmittance of $50.0 \%$.

In general, each dichroic pigment exhibited a different wavelength dependency of absorbance. Furthermore, the

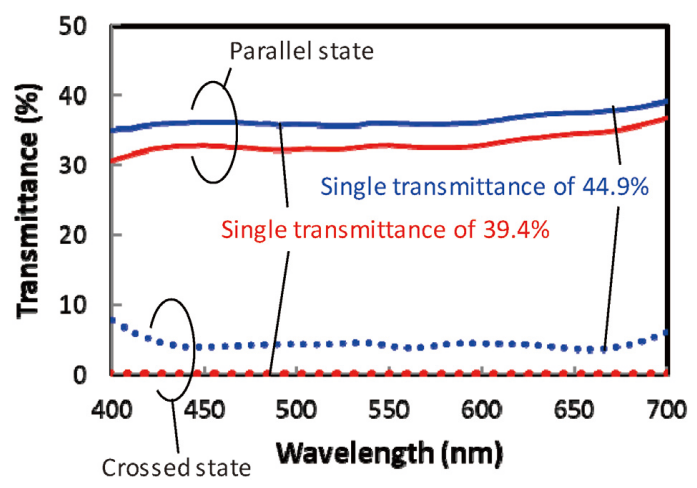

Fig.7 Wavelength dependency of our new achromatic polarizer in crossed and parallel states.

wavelength dependency of absorbance varies depending on the concentration. Therefore, it is necessary to optimize the composition balance of each dichroic pigment to achieve the desired transmittance at each wavelength.

From this result, we confirmed that it is possible to fabricate an achromatic polarizer with an arbitrary transmittance by controlling the concentration of dichroic pigments. Figure 7 shows the wavelength properties of our achromatic polarizers.

Figure 8 compares the chromaticity of polarizers in the parallel and crossed states and Fig.9 shows a photograph of the achromatic polarizer compared to a conventional iodine-based polarizer. The chromaticity of the conventional iodine-based polarizer is high, typically around $b^{*}=7.6$. In contrast, the chromaticity of our achromatic polarizer was within \pm 1.0 in the single, parallel and crossed states. Furthermore, we confirmed that our achromatic polarizer was a paper-like white color without wavelength dependence in the parallel state. These results show that the transmittance of our achromatic polarizer is constant in the visible wavelength range, and that our polarizer is achromatic with small values of $a^{*}$ and $b^{*}$ compared to conventional high-contrast and paper-white iodine-based polarizers. 


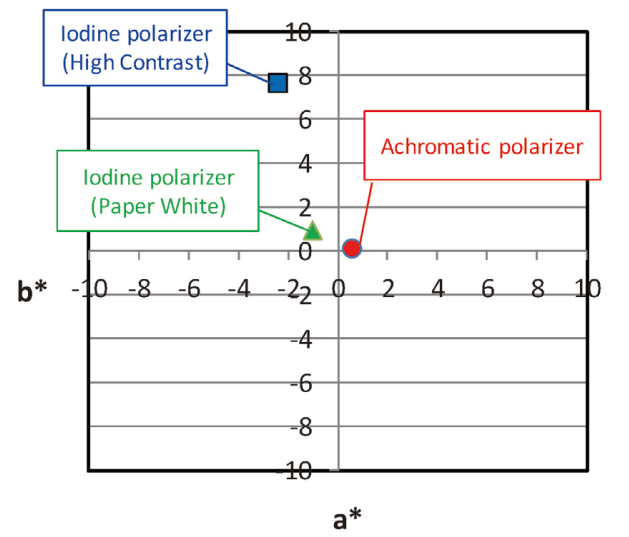

(a)

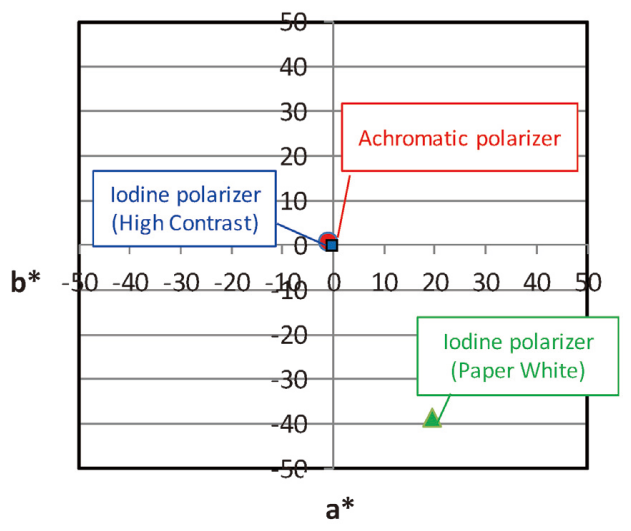

(b)

Fig.8 Comparison of chromaticity of polarizers in (a) parallel and (b) crossed states.

\section{Fabrication of reflective LCD using achromatic polarizer}

We fabricated a monochrome-type twisted nematic (TN)-mode reflective LCD and evaluated the reflectivity and chromaticity in the bright state. The fabrication process of the reflective TN-mode LCD was as follows. We coated an alignment layer (AL1254; JSR Corp.) over the glass substrate and baked at $200^{\circ} \mathrm{C}$ for $1 \mathrm{~h}$, followed by a rubbing treatment. After spraying of spacer particles and sealing of both substrates, LC material (TD-1016XX; JNC) was injected by capillary action. Finally, we laminated the achromatic polarizer (single transmittance: $44.92 \%$, degree of polarization: $87.45 \%$ ), a light diffusion film ${ }^{26-32)}$ and a reflective polarizer (see Fig.10). We also prepared a TN-mode LCD using a conventional iodine-based polarizer (paper-white type) and a commercialized electrophoretic display (Kindle; Amazon) for comparison. We measured the reflectivity and chromaticity of the TN-mode LCD with a spectroradiometer (SR-UL1; TOPCON) from the normal direction of the reflective LCD. The incident angle of light was $30^{\circ}$ and we used a halogen lamp as a light

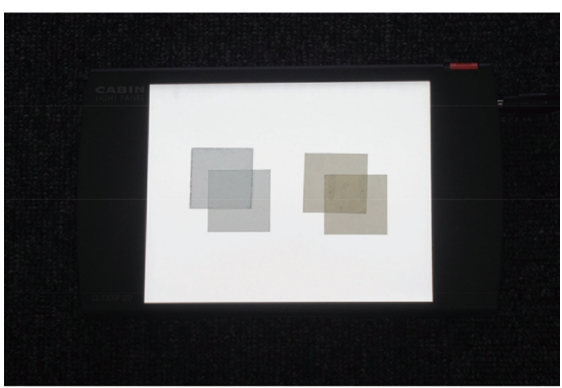

(a)

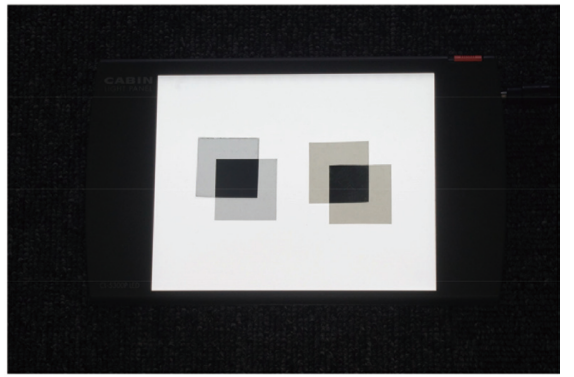

(b)

Fig.9 Photo of the polarizers in (a) parallel state and (b) crossed state. Left, Achromatic polarizer; Right, Iodine-based polarizer.

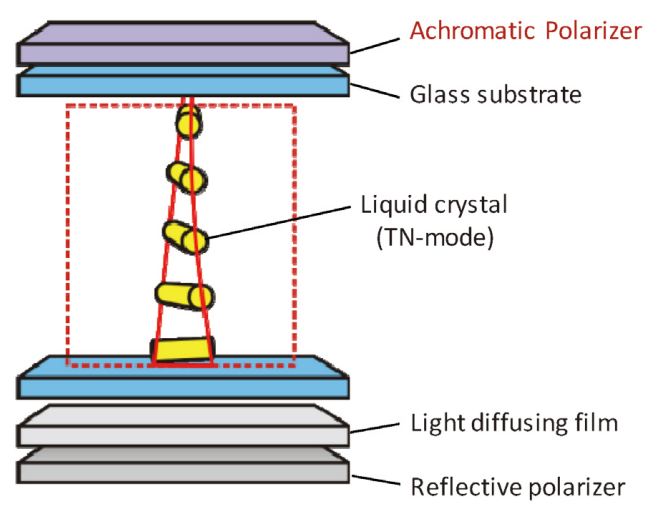

Fig.10 Configuration of the monochrome-type TN-mode reflective LCD using achromatic polarizer.

source.

Measurement results of reflectivity and chromaticity for our reflective $\mathrm{LCD}$, and a photograph of the monochrome-type reflective LCDs, are shown in Fig.11 and Fig.12, respectively. In general, an electrophoretic display has high reflectivity and high achromaticity due to light diffusion by charged pigment particles without polarizers. We found that our reflective LCD had a high reflectivity of $\mathrm{L}^{*}=71$ and a high achromaticity superior to the electrophoretic display, and confirmed that we successfully realized a high quality reflective LCD using our achromatic polarizer with high transmittance.

\section{Conclusion}

We have succeeded in the development of an achromatic polarizer, with no wavelength dependency in 


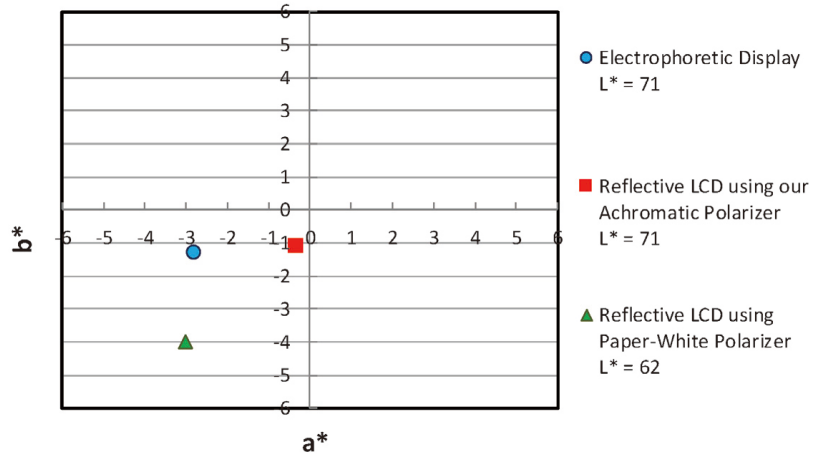

Fig.11 Reflectivity and chromaticity of reflective LCDs in bright state.

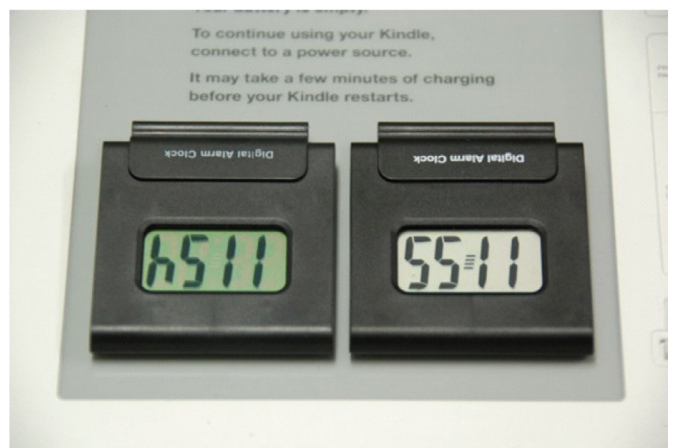

Fig.12 Photograph of the monochrome-type reflective LCDs. Left, conventional reflective LCD; right, our prototype; back, commercial electrophoretic display.

both the parallel and crossed states, using new dichroic pigments and a novel manufacturing process. We synthesized new dichroic pigments that suppress light absorption occurring in wavelength ranges outside the dominant wavelength, with the same dichroic ratio at the dominant wavelength. In addition, we established a new technique for impregnation of dichroic pigment and crosslinker (boric acid) and optimized the stretching conditions to control the alignment direction of each dichroic pigment.

We successfully developed a monochrome-type reflective LCD using our achromatic polarizer and achieved a high reflectance of $\mathrm{L}^{*}=71$, paper white characteristics superior to a conventional electrophoretic display and motion picture capabilities.

Our reflective display is therefore suitable for both small and large size display applications, including ebook readers, electronic shelf-edge labels (SELs) and digital signage. In addition, our new achromatic polarizer can be used in circular polarizers for antireflection of organic light-emitting diodes (OLEDs). Therefore, this newly developed achromatic polarizer will have a significant impact on future low-power display systems.

\section{References}

1) Y. Fukunaga, M. Tamaki, M. Mitsui, K. Maeda, M. Kabe, Y. Teranishi, T. Nakanishi, H. Omori, S. Hayashi, N. Takasaki, F. Goto, A. Higashi, T. Harada and Y. Nakajima: "Low Power, High Image Quality Color Reflective LCDs Realized by Memory-in-Pixel Technology and Optical Optimization Using Newly-Developed Scattering Layer", SID Symposium Digest, pp.701-704 (2013)

2) M. Mitsui, Y. Fukunaga, M. Tamaki, A. Sakaigawa, T. Harada, N. Takasaki, T. Nakamura, Y. Aoki, T. Tsunashima, H. Hayashi and T. Nagatsuma: "High Image Quality Reflective Color LCD Using Novel RGBW Technology", SID Symposium Digest, pp.93-96 (2014)

3) D. Kubota, Y. Niikura, R. Hatsumi, T. Ishitani, Y. Hirakata, H. Miyake, S. Yamazaki, A. Nakamura, Y. Chubachi and M. Katayama: "Reflective LCD with High Reflectance and Color Reproductivity for Reduced Eye Strain", SID Symposium Digest, pp.1084-1087 (2015)

4) T. Uchida, T. Katagishi, M. Onodera and Y. Shibata: Trans, IEEE, Electron Devices, ED-33, p. 1207 (1986)

5) N. Sugiura, K. Ohgiichi and T. Uchida: "Modeling of Property of reflector for reflective color LCDs", Proc. of AM-LCD '94, pp.92-95 (1994)

6) N. Sugiura and T. Uchida: "Optimization of property of reflector for reflective color LCDs", Proc. of AM-LCD '95, pp.153-156 (1995)

7) Y. Itoh, N. Kimura, Y. Ishii, F. Funada and K. Awane: "Development of 512-color-reflective TFT-LCD", Proc. of AM-LCD '95, pp.409-410 (1996)

8) K. Tsuda, N. Kimura, S. Mizushima: "Development of Reflector for HR-TFTs", Sharp Technical Journal, pp.1-5 (1999)

9) I. Washizuka, K. Tsuda and M. Hijikigawa: "Design of Reflector with Rough Surface for Reflective Type Color LCD", Journal of the surface finishing society of Japan, 50, pp.621-624 (1999)

10) S. Mitsui, Y. Shimada, K. Yamamoto, T. takamatsu, N. Kimura, S. Kozaki, S. Ogawa, H. Morimoto, M. Matsuura and K. Awane: SID Symposium Digest, p. 437 (1992)

11) Y. Ishii, N. Kimura, F. Funada and K. Awane, "Trend in the development of low power color LCDs for personal information tools", Proc. of Euro Display '96, pp.115-118 (1996)

12) T. Ishinabe, T. Uchida, M. Suzuki and K. Saito: "A Bright Full Color Reflective LCD using Optically Compensated Bend cell (ROCB cell) with Fast Response", Proc. of Euro Display '96, pp.119122 (1996)

13) T. Ishinabe, T. Miyashita and T. Uchida: "Optical Design of R-OCB Mode Full-Color Reflective LCD with Wide Viewing Angle and High Contrast", Journal of the Society for Information Display, pp.243-246 (1998)

14) M. Shibazaki, T. Ishinabe, T. Miyashita, T. Uchida, M. Miyai, K. Yoshida and Y. Ugai: "Full-color reflective TFT-LCD using the ROCB mode with wide viewing angle and high contrast", Proc. of AM-LCD '98, pp.27-30 (1998)

15) T. Uchida, T. Ishinabe, T. Nakayama, M. Suzuki and T. Miyashita: "A Novel Reflective Liquid Crystal Display with High Resolution and Full Color Capability", Japanese Journal of Applied Physics, 43, No.12, pp.8094-8100 (2004)

16) T. Ishinabe and T. Uchida: "A Bright and Wide Color Gamut Reflective Full-color LCD using Diffused Light Control Technology", Proc. of the 8th IMID association with IDMC 2008 and Asia Display 2008, pp.1377-1380 (2008)

17) Takahiro Ishinabe, Kentaro Kusama, Satoru Shoshi, Hideo Fujikake: "Wide-Color-Gamut Reflective Color LCDs using DoubleLayered Directional Light Diffusion Film", ITE Transactions on Media Technology and Applications (MTA), 4, 1, pp.34-40 (2016)

18) T. Yokoyama et al.: Bell. Chem. Soc. Jpn., 68, p. 469 (1995)

19) Fumio Ide et al.: The Optical Films for Flat Panel Displays, pp.5390 (2004)

20) Jae Bok Chang, Jong Hak Hwang, Jong S. Park, Jae Pil Kim, Dyes and Pigments, Volume 88, Issue 3, Pages 366-371 (2011) 
21) Yutaka KAYANE, Kazuya OGINO, Yoshiteru OHTA, Toru ASHIDA, Toshihiko TANAKA, Sumitomo Chemical R\&D Reports, Vol 2002, Pages 23-30 (2002)

22) Miyazaki, T.; Hoshiko, A.; Akasaka, M.; Shintani, T.; Sakurai, S. Macromolecules 2006, 39(2921)

23) Miyazaki, T.; Hoshiko, A.; Akasaka, M.; Sakai, M.; Takeda, Y; Sakurai, S. Macromolecules 2007, 40(8277)

24) Takahiko Itou, Hideyuki Kitai, Akira Shimazu, Tsukasa Miyazaki and Kohji Tashiro, J. Phys. Chem. B, 2014, 118 (22), pp 6032-6037

25) Dong Hyun Song, Hyun Young Yoo, Jung Jin Lee and Jae Pil Kim, Molecular Crystals and Liquid Crystals, Volume 445, 2006 - Issue 1

26) K. Kusama, B. Katagiri, T. Orui and S. Shoshi, "Light-Diffusing Films Using Two-step UV Irradiation for Various Displays", 2013 SID International Symposium Digest, pp.1177-1180 (2013)

27) T. Okita, K. Kawamura, T. Ohno, M. Ueda, S. Kitayama, S Hozumi, "A Polymer Film That Controls Light Transmission LUMISTY-", Sumitomo Kagaku K K, pp.37-48 (1991)

28) K. Maeda, S. Ishizuka, T. Tsujino, H. Yamamoto and A. Takigawa: SPIE Vol.1536 Optical Materials Technology for Energy Efficiency and Solar Energy Conversion X, p.138 (1991)

29) M. Honda, S. Hozumi and S. Kitayama: 3rd Pasific Polymer Conference, p.159-169 (1993)

30) M. Nishizawa, K. Kusama, K. Sekiya, B. Katagiri, T. Kawakami and T. Uchida: "Investigation of Novel Diffuser Films for 2D LightDistribution Control", Proc. of the 18th International Display Workshops, pp.1385-1388 (2011)

31) M. Nishizawa, K. Sekiya, T. Kawakami, T. UCHIDA: "Synthesis and Optical Property Analysis of Novel Anisotropic Light Diffusing Film", Bulletin of the Chemical Society of Japan (2012), 85, 839-841

32) M. Nishizawa, K. Kusama, K. Sekiya, B. Katagiri, T. Kawakami, T. Uchida: "Novel Diffuser Films for 2D Light-Distribution Control", ITE Technical Report, 36, pp.9-15 (2012)

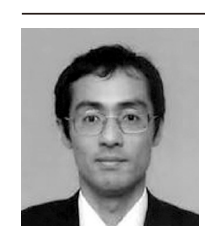

Noriaki Mochizuki received B.E. and M.E. degree from Yamanashi University, Kofu, Japan, in 2000 and 2002, respectively. He has joined Nippon Kayaku Co., Ltd. since 2002.

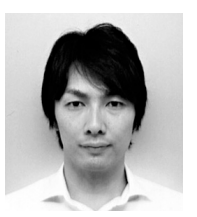

Takahiro Ishinabe received his B.S., M.S., and $\mathrm{Ph}$. D. degrees in Electronic Engineering from Tohoku University, Sendai, Japan, in 1995, 1997 and 2000, respectively. From 2000 to 2002, he was a Research Fellow of the Japan Society for the Promotion of Science and from 2003 to 2012, he was an Assistant Professor, and since 2013, he has been an Associate Professor in the Department of Electronics, Graduate school of Engineering, Tohoku University. He has also been a Visiting Professor in the CREOL, The College of Optics and Photonics, University of Central Florida from 2010 to 2011 . He has been performing a research on advanced liquid crystal displays such as wide viewing angle $L C D$, reflective full-color $L C D$, field sequential color LCD and flexible LCD.

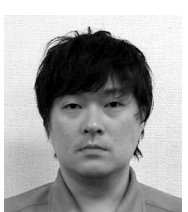

Daichi Fujiwara received B.E and M.E degrees from Yamagata University, Yamagata Japan in 2011 and 2013 respectively. He have joined Polatechno Co., Ltd. since 2013.

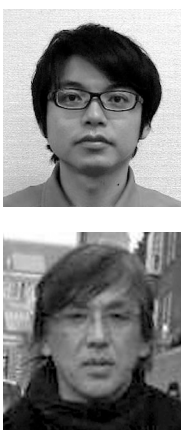

Daisuke Nakamura received B.E and M.E degrees from Niigata University, Niigata Japan in 2004 and 2006 respectively. He have joined Polatechno Co., Ltd. since 2006.

Norio Koma received Ph.D. degrees from Tohoku University, Japan, in 2002. He joined Sanyo Electronic Company in 1986-1999, 2002-2011. He have joined Polatechno Co., Ltd. since 2011. He received Best Poster Paper Award (Honorable Mention) from Society of Information Display (SID) in 1996.

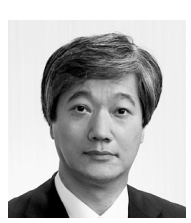

Hideo Fujikake received M.E and Ph.D. degrees from Tohoku University, Japan, in 1985 and 2003, respectively. In 1985, he joined Japan Broadcasting Corporation (NHK). In 1988-2012, he was with NHK Science and Technology Research Laboratories. Since 2012, he has been a professor at Department of Electronic Engineering, Tohoku University. He received Best Paper Award from Institute of Electronics, Information and Communication Engineers (IEICE) in 2001, Best Paper Award from Japanese Liquid Crystal Society (JLCS) in 2001 and 2015, Niwa-Takayanagi Best Paper Awards from Institute of Image Information and Television Engineers of Japan (ITE) in 2003 and 2009, and Electronics Society Award from IEICE in 2013. His current interest is concerned with flexible liquid crystal displays. He also served as a General Vice Chair in International Display Workshops in 2015-2016, a Japan Chapter Chair in IEEE Consumer Electronics Society in 2012-2014, and a Vice President of Japanese Liquid Crystal Society in 2015-2016. He is an IEICE fellow since 2015, and ITE fellow since 2016. 